\title{
Selbstkonfiguration und automatisiertes Änderungsmanagement von MES-Systemen
}

\author{
Senad Bukva, Udo Enste und Felix Uecker, LeiKon GmbH
}

\begin{abstract}
Anwendungen, die der MES-Ebene zugeordnet werden, eröffnen das Potential, erhöhte Wertschöpfungen aus Produktionsprozessen und der Anlagenbewirtschaftung zu erzielen. Ein Haupthindernis zur Nutzung dieser Anwendungen ist jedoch oftmals der Aufwand für deren Inbetriebnahme und dauerhaften Pflege. Ziel muss es daher sein, diesen Aufwand zu senken. Dieser Beitrag behandelt dazu konzeptionelle Voraussetzungen zur Nutzung von Automatismen der Systemintegration und Anwendungsbeispiele selbstkonfigurierender MES-Anwendungen aus der industriellen Praxis.
\end{abstract}

MES / Selbstkonfiguration / CPM / Alarm Management / Betriebsdatenerfassung / Asset Management Self Configuration and Automated Management of Change of
MES Applications

MES applications provide the chance to increase the benefit and the current yield of manufacturing processes. One obstacle to use these applications is the expected total cost of configuration and life cycle maintenance. An important goal is to minimize these efforts. This paper discusses the prerequisites to use self configuring MES applications and describes several implementations of self configuring MES solutions in practice.

MES / Self Configuration / CPM / Alarm-Management / PIMS / Asset Management

\section{Motivation}

Anlagen der Prozessindustrie werden heute überwiegend mit modernen Prozessleitsystemen überwacht und gesteuert. Durch den vermehrten Bedarf, weitere Wertschöpfungspotentiale der Produktionsprozesse und der Anlagenbewirtschaftung zu nutzen, entsteht eine wachsende Nachfrage und ein zunehmendes Angebot an ergänzenden IT-Lösungen zur Produktivitätsanalyse und -optimierung bzw. der Weiterverarbeitung von Produktionsinformationen. Dazu zählen Anwendungen wie Betriebsdatenerfassungssysteme, Lösungen des Controller Performance Monitorings, der Gerätezustandsbeobachtung (Condition Monitoring), des Alarm-, Asset- und Instandhaltungsmanagements sowie Applikationen zur Qualitätssicherung des Engineerings von Leitsystemen (Leitsystemqualifizierung). Alles Lösungen, die in der Regel nicht integraler Bestandteil von Prozessleitsystemen, sondern oberhalb der Leitsystemebene anzuordnen sind und insgesamt einen heterogenen Verbund von Systemen unterschiedlicher Hersteller bilden. Im Folgenden werden diese vereinfacht als „ergänzende Anwendungen“ zu bereits im Einsatz befindlichen Leitsystemen bezeichnet. Klassischerweise handelt es sich um Anwendungen der Betriebs- und Unternehmensleitebene (siehe Ebenenmodell in [1]). Bezug nehmend auf internationale Standards (siehe z.B. [2]), ordnet man diese Anwendungen heute der so genannten MES-Ebene zu.

Trotz bedarfsgerechter Funktionalitäten und des unbestrittenen Nutzenpotentials werden ergänzende MES-
Anwendungen im betrieblichen Alltag noch nicht in dem Maße eingesetzt, wie es sowohl aus Betreiber- als auch aus Anbietersicht wünschenswert ist. Aus Sicht der Betreiber ist das Nutzen-Aufwands-Verhältnis mitunter noch nicht ausreichend groß, um Investitionen in ergänzende MES-Lösungen zu tätigen. Ein wichtiger Grund dafür ist deren Engineeringaufwand bei der Inbetriebnahme und vornehmlich in der dauerhaften Pflege. In vielen Produktionsbetrieben werden derartige Anwendungen oftmals noch gemieden, da die Betreiber den Aufwand höher einschätzen als den Nutzen bzw. den Aufwand, selbst bei Annahme eines langfristig positiven Aufwand/Nutzen-Verhältnisses, als momentan nicht leistbar betrachten. Potentiale der Wertschöpfung von Produktionsanlagen liegen damit brach. Aus Sicht der Systemanbieter bedeutet das im Gegenzug, dass das Marktpotential ergänzender MES-Lösungen nicht ausgeschöpft werden kann.

Aus dieser Motivation heraus wurden neue methodische Ansätze eingesetzt, um Lösungen zur Selbstkonfiguration für Systeme der MES-Ebene zu entwickeln. Der Ansatzpunkt basiert dabei auf zwei Aspekten:

- Konfigurationsinformationen, die zur Einrichtung und fortwährenden Nachpflege ergänzender IT-Lösungen der MES-Ebene benötigt werden, sind bereits überwiegend oder zum Teil sogar vollständig als Daten in geordneten Strukturen in Leitsystemen vorhanden.

- Moderne Datenkommunikationstechnologien ermöglichen über offene Leitsystemschnittstellen neben dem Auslesen von Datenwerten mittlerweile auch das Erkun- 
den von Engineeringdaten in systemunabhängiger Form (siehe [3]).

Daraus ergibt sich, dass externe Systeme den Datenhaushalt eines Leitsystems „abscannen“ können, um die für sie relevanten Konfigurationsdaten automatisch zu ermitteln. Das Leitsystem wird damit auch in heterogenen Systemverbünden zur "Single Source of Engineering" (siehe Bild 1).

In diesem Artikel werden Grundprinzipien der Selbstkonfiguration und deren Anwendung in der industriellen Praxis vorgestellt.

\section{Selbstkonfigurierende Systeme}

Ein technisches System wird als selbstkonfigurierend bezeichnet, wenn es in der Lage ist, sich selbst ohne externe Hilfe zu konfigurieren. In der Automatisierungstechnik findet man methodische Ansätze und Lösungen für selbstkonfigurierende Systeme in unterschiedlichen Anwendungsfeldern. Beispiele dazu sind:

- Selbstkonfigurierende Funknetze: Funkteilnehmer (z.B. Sensoren in Sensornetzwerken) suchen von sich aus geeignete Funkknoten und optimieren laufend selbstständig ihre Funkverbindungen (siehe u. a. [4]).

- Selbstkonfigurierende Betriebssysteme: Das Betriebssystem "Knoppix" (eine frei erhältliche GNU\Linux-Distribution) konfiguriert sich beim Start von einer Boot-CD selbst, indem es die zur gefundenen Hardware passenden Treiber lädt.

- Selbstkonfigurierende Asset Management Anwendungen: Unter Ausnutzung von Erkundungsdiensten und Profilfestlegungen von Profibus wurde ein feldnaher Informationsserver unter dem Namen "Asset Management Box" entwickelt, der eigenständig die Topologie und sämtliche Teilnehmer einer Feldbusinstallation identifiziert und unter anderem Diagnosedaten der angeschlossenen Geräte auswertet [5]. Andere Arbeiten beschreiben Ansätze zur Selbstkonfiguration von Asset Management Anwendungen basierend auf CAE-Daten (siehe $[6 ; 7 ; 8]$ ).

- Selbstkonfigurierende Visualisierungen, bei der auf der Basis elektronischer Gerätebeschreibungen oder Funktionsbausteindaten Visualisierungskomponenten für Bedien- und Beobachtungsoberflächen generiert werden (siehe [9; 10]).

Gemeinsam ist allen Ansätzen, dass Datenhaushalte bestehender Systeme in Form von Regeln und daraus resultierenden Abfolgen von Kommunikationsdiensten durchsucht werden, um die Konfiguration einer externen Anwendung automatisch zu extrahieren. Voraussetzung dazu ist, dass innerhalb einer Anwendungsdomäne ein gemeinsames Verständnis von Grundstrukturen existiert, das in überlagerten Datenmodellen (Metamodellen) abbildbar ist. Dies ist notwendig, um zum einen die spezifischen Daten eines Quellsystems in eine vereinheitlichte Semantik zu überführen und in Form vereinheitlichter Syntaxvorgaben anderen Systemen zur Verfügung zu stellen. Zum anderen ist ein

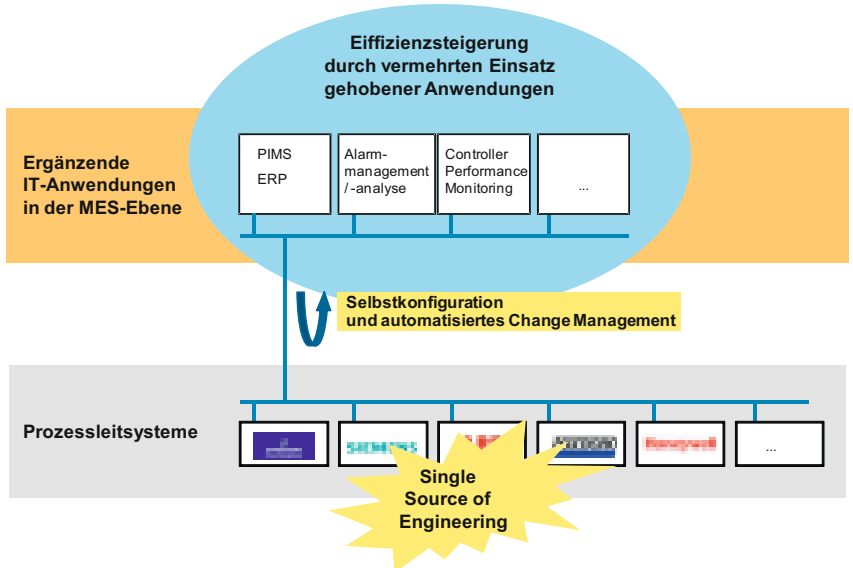

Bild 1: Selbstkonfiguration und automatisiertes Änderungsmanagement von Anwendungen der MES-Ebene.

Metamodell notwendig, um allgemeingültige, systemunabhängige Regeln zum Suchen relevanter Daten zu formulieren. Ein typisches Metamodell der Leittechnik ist das Funktionsbausteinmodell. Jedes Prozessleitsystem ermöglicht die Umsetzung von Regelungs- und Steuerungslogiken in Form von Funktionsbausteinnetzen, deren Funktionsbausteine von bestimmten Bausteintypen abgeleitet werden. Die Bausteintypen werden über Schnittstellendefinitionen (Angabe von Ein- und Ausgangsparametern) und einer Bausteinmethode definiert. Über Verbindungen von Parametern unterschiedlicher Bausteininstanzen können Bausteinnetzwerke gebildet werden, die zusammen die gewünschten Regelungs- und Steuerungslogiken bilden. Dieses herstellerübergreifend geltende Metamodell der Bausteintechnik ist damit ein Beispiel innerhalb einer spezifischen Anwendungsdomäne (hier: Steuerungsstrukturen in Leitsystemen).

Übersichten über den Nutzen und die Notwendigkeit von Metamodellen finden sich in [11] am Beispiel des automatisierten Austauschs von Anlagendaten in CAE-Systemen und in [12] für die Anwendung generischer Datenkommunikationssysteme.

\section{Steigerung der Wertschöpfung}

Als Ziele einer Effizienzsteigerung der Produktionsverfahren und der Anlagenbewirtschaftung stehen bei Anlagenbetreibern folgende Arbeitsfelder im Fokus:

- Steigerung der Anlagenverfügbarkeit und des Durchsatzes

- Zunahme der Material- und Energieeffizienz

- Verbesserung des Instandhaltungs- und Qualitätsmanagements

- Erhöhung der Umweltverträglichkeit.

Durch sukzessive Modernisierungen und Erweiterungen der Prozessleitsysteme kann der Automatisierungsgrad erhöht und damit in der Regel auch die Wertschöpfung in den angegebenen Feldern gesteigert werden. Möchte man darüber hinaus die Wertschöpfung weiter steigern, bietet es sich an, ergänzende Anwendungen oberhalb des Prozessleitsystems zu nutzen. Bild 2 soll diesen Effekt anhand eines Polardiagramms verdeutlichen. 


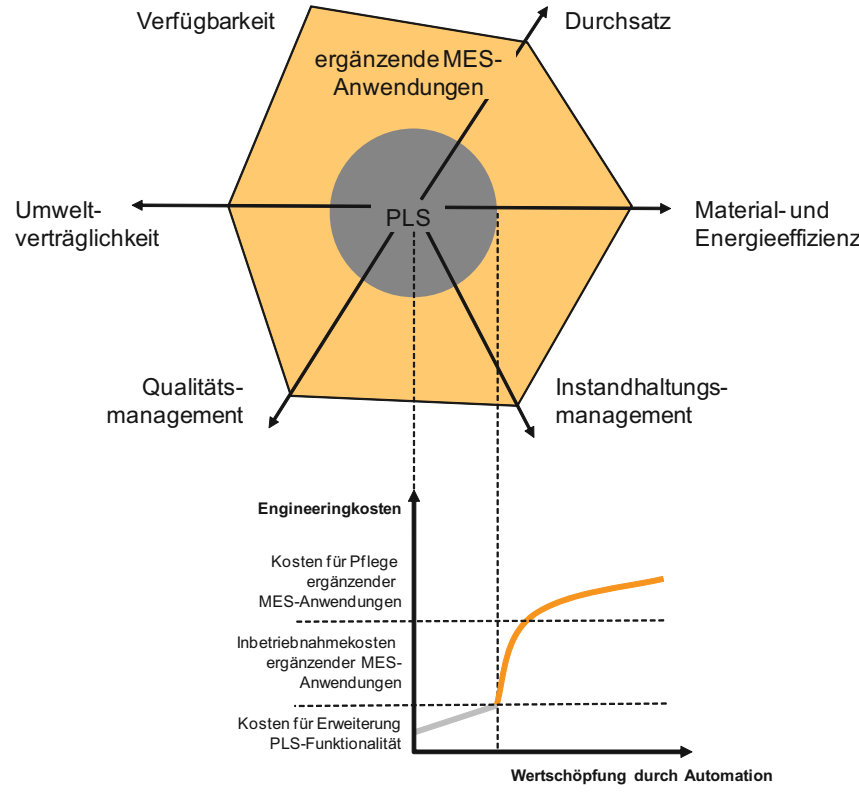

Bild 2: Wertschöpfung durch Automation.

Um den Verbesserungszielen näher zu kommen, steht im Kern die möglichst hohe Ausnutzung der Funktionalitäten eines Prozessleitsystems (graue Fläche im Polardiagramm). Wenn der Funktionsumfang eines Leitsystems an seine Grenzen stößt (und damit der graue Kern nicht mehr erweiterbar ist), können ergänzende MES-Anwendungen eingesetzt werden, die zur weiteren Annäherung an einzelne Ziele, wie z. B. der Verbesserung der Material- und Energieeffizienz, dienen (orangefarbene Fläche im Polardiagramm).

Erweitert man das Polardiagramm um eine dritte Dimension, die die Engineeringkosten qualitativ darstellt (siehe Bild 2 unten), erkennt man, dass Kosten für PLS-Erweiterungen in der Regel direkt einhergehen mit Wertschöpfungsgewinnen (hier im grauen Kurvenabschnitt vereinfacht linear dargestellt). Der Einsatz neuer, ergänzender Anwendungen bedingt jedoch, dass bei deren Inbetriebnahme die Engineeringkosten steigen, ohne dass zunächst ein signifikanter Wertschöpfungsgewinn erzielt wird. Erst wenn die Systeme eingerichtet sind, ergeben sich wieder positive Relationen zwischen Aufwand und Nutzen. Der gesamte Aufwand, bis die Tangente

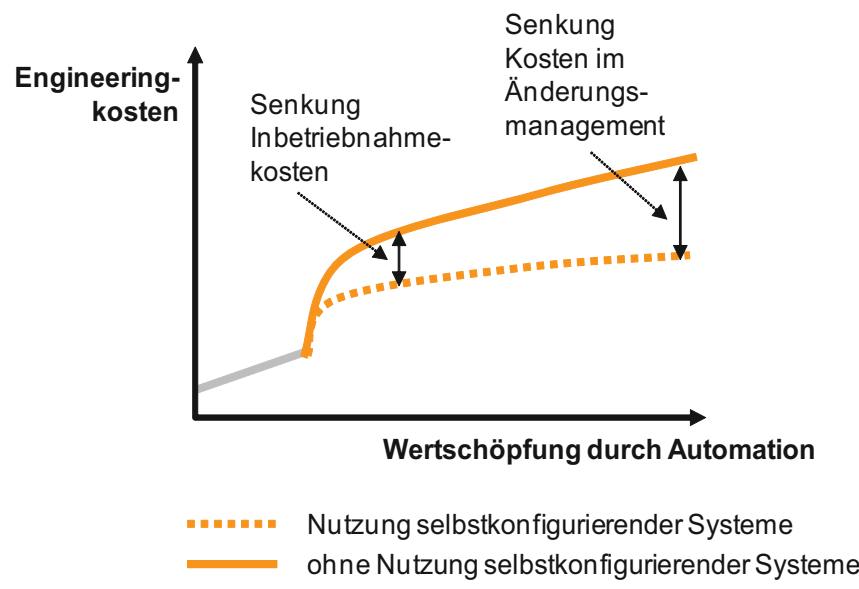

Bild 3: Kostenreduktion bei Nutzung selbstkonfigurierender Systeme. des Kurvenverlaufs nach Einführung eines neuen Systems wieder eine bestimmte Steigung unterschreitet, kann zu den Inbetriebnahmekosten gezählt werden. Die Steigung, die die Kurve nach der Inbetriebnahme zeigt, repräsentiert die laufenden Kosten für das Änderungsmanagement. Damit Investitionen in ergänzende MES-Anwendungen überhaupt getätigt werden, darf zum einen der Anstieg des Kostenkurvenverlaufs in der Einführungsphase eine vorgegebene Investitionssumme nicht überschreiten und zum anderen sollte die Steigung des Kostenverlaufs, die durch Pflege- und Erweiterungsaufwendungen der MES-Lösungen bestimmt wird, möglichst flach verlaufen.

Eine Investitionsentscheidung zum Einsatz ergänzender MES-Lösungen hängt demnach wesentlich davon ab, welchen Engineeringaufwand sie bei der Inbetriebnahme und vornehmlich in der dauerhaften Pflege mit sich bringt. Eine Investition in ergänzende MES-Anwendungen kommt oftmals nicht zustande, da der von Budgetverantwortlichen vorgegebene Zeitraum des "Return of Invest" (ROI) nicht ausreicht, um in dieser Zeitspanne einen genügend großen Wertschöpfungsgewinn im Vergleich zu den bis dahin auflaufenden Kosten zu erzielen. Ein unbefriedigendes Ergebnis sowohl für Systemanbieter als auch für Anlagenbetreiber.

Um eine positive Investitionsentscheidung herbeiführen zu können, muss der Aufwand zur Einrichtung und laufenden Konfigurationspflege verringert oder im Idealfall eliminiert werden. Durch die sukzessive Nutzung von Automatismen der Systemkonfiguration wird erwartet, dass eine wichtige Hürde für den Einsatz ergänzender MES-Lösungen zur Optimierung der Produktion herabgesenkt wird. Bild 3 zeigt den zu erwartenden Effekt. Bei Einführung ergänzender MESAnwendungen kann dabei der sprunghafte Kostenanstieg gesenkt und im Laufe des Lebenszyklus derartiger Anwendungen können die variablen Kosten und damit die Steigung der Kostenkurve verringert werden. Damit entsteht eine WinWin-Situation für Anwender und Systemanbieter.

Konfigurationsdatenkoppler, die eine Selbstkonfiguration und einen fortlaufenden Konsistenzabgleich von Engineeringdaten unterschiedlicher Systeme durchführen, müssen dabei nicht integraler Bestandteil einer ergänzenden MESLösung oder des Prozessleitsystems sein. Stattdessen können Importschnittstellen oder Programmierschnittstellen, die alle gängigen MES-Lösungen in unterschiedlichster Form anbieten, von externen Engineering-Datenkopplern genutzt werden. Insgesamt werden so die variablen Kosten für die Inbetriebnahme und für die laufende Pflege ergänzender Anwendungen der MES-Ebene vermindert.

\section{Geeignete Kommunikationstechnologien}

Der Ansatz der Selbstkonfiguration basiert auf dem Grundgedanken, dass die überwiegende Anzahl an Informationen, die zur Konfiguration ergänzender Lösungen der MES-Ebene benötigt werden, bereits in strukturierter Form in den Leitsystemen hinterlegt ist. Über vereinheitlichte, offene Schnittstellen können diese Informationen mit Hilfe regelbasierter Konfigurationskoppler gesucht und ausgelesen werden, so dass die überlagerten Anwendungen „auf Knopfdruck“ in 
Betrieb genommen werden können und das Engineering auf das Prozessleitsystem beschränkt bleibt.

Damit Lösungen der Selbstkonfiguration breit angewendet werden können, sollten standardisierte Kommunikationsprotokolle verwendet werden. Damit wird gewährleistet, dass Suchregeln über vereinheitlichte Kommunikationsdienste systemunabhängig realisierbar sind. Die Anforderungen der Selbstkonfiguration verteilter Systeme erfordern so genannte "Whitebox"-Kommunikationstechnologien. Diese ermöglichen es, die den einzelnen Datenhaushalten unterlagerten Datenmodelle transparent zu machen und Datenobjektklassen, Datenobjekteigenschaften und die typischerweise vorliegenden netzartigen Datenbeziehungsstrukturen auf allgemeingültige Kommunikationsobjekte abzubilden. Darüber hinaus müssen die Kommunikationsprotokolle vereinheitlichte Erkundungsdienste anbieten, um die Möglichkeit zu eröffnen, Metainformationen auch ohne Vorabwissen finden zu können.

Eine Analyse heute verfügbarer Kommunikationstechnologien zeigt, dass nur wenige von ihnen diesen Anforderungen genügen [3]. Kommunikationstechnologien, die die Nutzung der Selbstkonfiguration unterstützen, findet man in zwei Kategorien:

- Durch die heutigen nicht mehr streng hierarchisch organisierten Systemtopologien ergibt sich die Möglichkeit, dass Anwendungen der MES-Ebene direkt als Master an einen Feldbus angeschlossen werden können. Diese Vorgehensweise bietet sich insbesondere für Anwendungen des Asset- und Instandhaltungsmanagements an. In der Kategorie der Feldbustechnologien bieten Profibus und Foundation Fieldbus Mechanismen, selbstkonfigurierende Systeme zu realisieren. Ein typischer Dienst, der in beiden Technologien für Selbstkonfigurationszwecke externer Anwendungen genutzt werden kann, ist der Live-List-Dienst, mit dem die Adressen momentan installierter Geräte in einem Feldbussegment ermittelt werden können. Entscheidende Grundlage für weiterreichende Selbstkonfigurationen auf Feldebene sind Profildefinitionen einzelner Geräteklassen, wie sie insbesondere für Profibus bzw. Profinet bereits ausgearbeitet wurden.

- Zwischen Prozess- und MES-Ebene stehen als Kommunikationstechnologien im Bereich der Leittechnik Webservices, der offene Kommunikationsstandard ACPLT/KS [13] und zukünftig auch OPC UA [14] zur Verfügung, die grundlegende Bedingungen zur Umsetzung selbstkonfigurierender Systeme unterstützen. Der Einsatz von Webservices für Selbstkonfigurationsfunktionen hängt dabei stark von der Vereinheitlichung von XML-Schemata ab, die pro Anwendungsdomäne definiert sein müssen. ACPLT/KS ist ein seit mehreren Jahren verfügbarer Standard, mit Hilfe dessen bereits zahlreiche selbstkonfigurierende MES-Systemankopplungen umgesetzt wurden. Der Standard OPC UA bietet einen vergleichbaren Datenobjekt- und Diensteumfang wie ACPLT/KS. Für diesen Standard sind derzeitig aber noch keine Leitsystemschnittstellen am Markt verfügbar. Durch den hohen Grad der Datenmodell- und Funktionsüberlappung von ACPLT/KS und OPC UA ist es heute schon sinnvoll, Anwendungen via ACPLT/KS zu realisieren, um diese später mit geringem Aufwand nach OPC
UA zu migrieren, sobald geeignete Schnittstellen für diese neue Technologie zur Verfügung stehen.

Die aufgeführten Kommunikationstechnologien bieten die Möglichkeit, systemspezifische Datenobjekte direkt in der Schnittstelle des Systems auf generische Datenobjekte abzubilden. Damit wird die Umsetzung der Suchregeln systemunabhängig und robust gegenüber internen Änderungen der Datenverwaltung bei Updates und Upgrades der Systeme. Eventuell nötige Änderungen konzentrieren sich dabei ausschließlich auf eine Stelle, nämlich der Anpassung von internen Aufruffunktionen innerhalb der Schnittstelle. Die überlagerten Anwendungen können von Änderungen abgeschirmt werden. Alternative, in der Praxis häufig vorkommende Lösungsformen, basieren darauf, dass bei Konfigurationsdatenauslesungen direkt auf die Engineeringdatenbanken zugegriffen wird (z.B. über spezifische ODBCQueries) oder Suchregeln über zuvor festgelegte Namenskonventionen durchgeführt werden (z.B. via OPC DA). Auch hierüber sind Selbstkonfigurationslösungen möglich, diese führen in der Regel aber zu höherem Aufwand im Änderungsmanagement, sowohl bei der Verbreitung selbstkonfigurierender Ankopplungen auf andere Leitsysteme als auch bei nötigen Anpassungen der Selbstkonfigurationsfunktionen bei Updates und Upgrades.

\section{Anwendungsbeispiele}

Die Anwendungsbeispiele selbstkonfigurierender Systeme umfassen ein breites Spektrum an Lösungen der MES-Ebene. Zum einen betreffen sie bereits bestehende Systemlösungen am Markt, die heute noch einen manuellen Konfigurationsvorgang benötigen, der durch einen voll- oder zumindest semiautomatischen Konfigurationsvorgang ersetzt werden kann. Zum anderen eröffnen die Möglichkeiten einer offenen Konfigurationsdatenerkundung auch die Entwicklung neuartiger Anwendungen insbesondere im Bereich des Asset Managements und der Leitsystemqualifizierung.

\subsection{Controller Performance Monitoring}

Anwendungen des Controller Performance Monitorings (CPM) dienen der kontinuierlichen Performancebewertung von Regelkreisen. Dazu lesen sie über Leitsystemschnittstellen in zyklischen Abständen pro Regler Signalsequenzen der drei Parameter Sollwert, Istwert und Reglerausgang aus und bewerten damit den Regelkreis anhand regelungstechnischer KPIs (Key Performance Indicators). So können beispielsweise schlechte Regelgüten (starke Abweichungen von Soll- und Istwert), Oszillationsverhalten, Dimensionierungsprobleme oder auch unzureichende Wirkeinflüsse der Stellorgane detektiert werden. Darauf aufbauend können über Wissensbasen textuelle Hinweise für das Betriebspersonal generiert werden, die anzeigen, wo mit hoher Wahrscheinlichkeit eine Problemursache zu finden ist.

Zur Inbetriebnahme von Systemen des Controller Performance Monitorings müssen für jeden Regelkreis Engineeringdaten konfiguriert werden. Dazu zählen: 
- die Konfigurationsnamen der einzelnen Regler

- die Adressierungspfade aller kontinuierlich einzulesenden Reglersignale

- die im Prozessleitsystem eingetragenen Mess- bzw. Stellbereiche der einzelnen Regler inkl. der Einheitenbezeichnungen der dazugehörigen Signale

- ggf. die aktuell eingestellten PID-Reglerparameter

- ggf. Zusatzinformationen, ob der Regelkreis z.B. im "Reversed Mode" betrieben wird oder ein "Anti Windup" aktiviert wurde.

Wie leicht zu erkennen ist, sind diese Informationen herstellerübergreifend über die im Leitsystem instantiierten Funktionsbausteine vom Typ „Regler" abgreifbar. Das heißt, über eine Suche von aktuell instantiierten Funktionsbausteinen vom Typ „Regler" kann eine CPM-Anwendung sämtliche der oben genannten Konfigurationsdaten aus einem Leitsystem ermitteln, ohne dass dieser Anwendung explizit bekannt gemacht werden muss, welche Regler im Leitsystem eingerichtet sind (s. Bild 4).

Eine derartige Selbstkonfiguration ist beispielsweise für die PCT Optimizer Suite der Firma PCT Technology AB verfügbar. Diesem CPM-System muss lediglich mitgeteilt werden, um welchen Typ von Leitsystem es sich handelt, an das es angekoppelt wird und unter welcher IP-Adresse das Leitsystem kontaktiert werden muss. Unter der Funktion „Automatic Config" werden daraufhin sämtliche relevanten Engineeringdaten aus dem Leitsystem ausgelesen und die Performanceüberwachung kann direkt gestartet werden. Es konnte in industriellen Einsätzen gezeigt werden, dass die Inbetriebnahme einer Reglerperformanceüberwachung von bis zu 1000 Regelkreisen in weniger als einer Stunde möglich ist. Da sämtliche CPM-Anwendungen im Kern die glei-

\section{CPM-Tool}

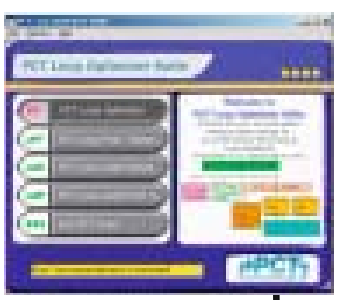

automatisierte Erkundung allerFunktionsbaustein-

instanzen vom Typ PID-Regler;

automatisiertes Auslesen

von Engineeringdaten

basierend auf

PID-Masterkonfiguration

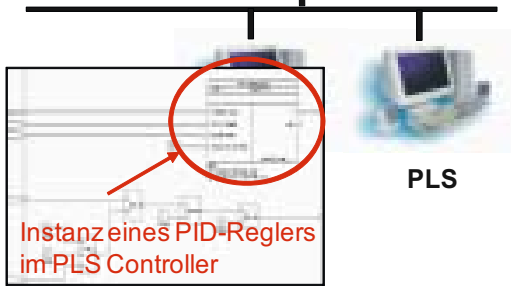

Bild 4: Selbstkonfiguration eines Controller Performance Monitoring Tools. chen Engineeringdaten benötigen, ist das Prinzip der Selbstkonfiguration grundsätzlich auch auf andere am Markt verfügbare CPM-Lösungen übertragbar.

\subsection{Alarmmanagement}

Alarmfluten beeinträchtigen in immer größerem Maß die Arbeit von Anlagenfahrern. Zur Verbesserung des Alarmmanagements können Alarmanalysen durchgeführt werden, um sich ein Bild über die Beanspruchung der Anlagenfahrer zu machen. Bei Alarmanalysen unterscheidet man zwischen statischen und dynamischen Analysen. Statische Analysen betrachten den Zustand der Alarmkonfigurationen. Dynamische Alarmanalysen untersuchen das Alarmauslöseverhalten im operativen Betrieb, insbesondere die Häufigkeiten von Alarmaktivierungen pro Zeiteinheit oder pro Alarmquelle (siehe u.a. [15]).

Konzentriert man sich zunächst auf die Optimierung der statischen Alarmkonfiguration, so können "Best Practice"Empfehlungen der britischen „Engineering Equipment and Materials Users Association“ (EEMUA) genutzt werden, die besagen, dass nicht mehr als $5 \%$ aller konfigurierten Alarme der höchsten Alarmpriorität zugeordnet werden sollten, damit Anlagenfahrer im Störungsfall eine reale Chance haben, sich auf die wichtigsten Alarme zu konzentrieren [16]. Zudem sollen Meldetexte eindeutig verwertbare Hinweise auf Problemursachen und möglichst Handlungsanweisungen beinhalten.

Eine derartige statische Alarmanalyse kann selbstkonfigurierend umgesetzt werden. Pro Leitsystem sind mögliche Alarmquellen und deren Prioritätseinstellungen bzw. aktuell konfigurierte Meldetexte über Suchregeln auffindbar. Bei den meisten Leitsystemen kann dies wiederum durch eine Suche nach Funktionsbausteinen bestimmter Typen realisiert werden, wobei aus der Dokumentation der Bausteine bekannt ist, welche Parameter zum Aufstellen der statischen Alarmanalyse auszulesen sind. Alternativ können Engineeringdatenbanken der Prozessleitsysteme erkundet werden. Das Ergebnis ist ein Analysevorgang, mit dem man mit geringem Aufwand die Abnahme von Leitsystemen und ein regelmäßiges Screening der statischen Alarmkonfiguration laufender Systeme durchführen kann. Dies ist insbesondere relevant, da Empfehlungen, wie in der "EEMUA"-Richtline beschrieben, als Stand des Wissens bewertet werden, mit dem Verantwortliche bei Untersuchungen nach Störfällen konfrontiert werden können.

\subsection{Zentralisierte Betriebsdatenerfassung}

Bedeutende Teile eines Prozessleitsystems sind das Prozesswert- und Meldearchiv. Diese Archive sind auf den Messstellenbestand und die Meldequellen der im Leitsystem integrierten Controller ausgelegt. Typischerweise ist ein Leitsystemarchiv daher einer bestimmten Anlage oder einem bestimmten Betrieb zugeordnet und wird primär von den jeweiligen Betriebsverantwortlichen und Anlagenfahrern genutzt. In der Regel werden im Leitsystemarchiv Daten über einen Zeitraum von 3 bis 12 Monaten vorgehalten. Behördliche Vorgaben für längerfristige Produktionsdaten- 
speicherungen insbesondere aber Anforderungen umfassenderer Produktionsdatenanalysen, -reportings und -optimierungen erfordern Langzeitarchive in Form zentralisierter Betriebsdatenerfassungssysteme. Hier werden Daten unterschiedlicher Anlagen aus den Leitsystemen ausgelesen und in einem zentralen Datenbestand zusammengeführt. Darauf aufbauend werden gehobene Verfahren der Datenanalyse und -aufbereitung angeboten. Die Konfiguration dieser so genannten „Plant Information Management Systeme" (PIMS) umfasst zu einem wesentlichen Teil die Einrichtung einzulesender Datenpunkte, deren Skalierungen, Einheiten und Komprimierungsfaktoren. Üblicherweise werden diese Daten heute manuell pro Messstelle konfiguriert. Als eine erste Hilfe nutzt man ggf. den Import von Excel-Datenblättern, so dass über leicht durchzuführende Kopiervorgänge ein Bulk-Engineering durchgeführt werden kann.

Ein zusätzliches, bei der zentralisierten Betriebsdatenerfassung immer wieder zu lösendes Problem ist das der Sicherstellung lückenloser Dateneinlesungen aus den Leitsystemen. Typischerweise werden Onlinekopplungen eingerichtet, bei denen zyklisch oder auch ereignisorientiert Daten aus den Leitsystemen eingelesen werden. Über Redundanzstrukturen der Einlesewege und/oder über explizit eingerichtete Datenzwischenspeicher wird versucht, eine Ausfallsicherheit zu gewährleisten.

Eine Selbstkonfiguration von Betriebsdatenerfassungssystemen kann über eine einfache Strategie umgesetzt werden, die zusätzlich die Problematik der Ausfallsicherheit löst. Statt einer Onlinekopplung wird eine Archiv-Archiv-Kopplung genutzt. Die Regel, mit der eine Selbstkonfiguration einer zentralisierten Datenerfassung umgesetzt werden kann, lautet: „Alles, was ein Betrieb für aufzeichnungswürdig hält - und deshalb im Leitsystemarchiv bereits gespeichert wird -, soll auch in einem Langzeitarchiv für Analysen und Datenaufbereitungen zur Verfügung stehen". Aus Sicht eines Betriebsdatenerfassungssystems kann mit dieser Strategie über eine offene Leitsystemschnittstelle laufend ausgelesen werden, welche Messstellen im Leitsystem archiviert werden und welche zusätzlichen Engineeringinformationen, wie z.B. Messbereich und Einheit, pro Archivmessstelle hinterlegt sind. Auf Basis dieser Information kann eine Selbstkonfiguration der nötigen Tabellen bzw. Datenrecords im PIMS erfolgen und eine laufende Konsistenzsicherung dieses Engineeringdatenbestandes automatisch durchgeführt werden. Wird im Leitsystem eine Messstelle im Archiv hinzugefügt, erscheint sie automatisch auch im PIMS. Die eigentliche Nutzdatenauskopplung erfolgt über zeitnahe parallel dazu ausgeführte zyklische Zeitreihenübertragungen aus dem Prozesswertarchiv. Da das Prozesswertarchiv bei diesem Konzept als systembedingt bereits vorhandener Datenpuffer genutzt wird, kann der Datenkoppler auch bei zeitweisen Kommunikationsunterbrechungen Daten ohne interne Modusumschaltungen der Einleseart (typisch: „Online“ „Archive Recovery" oder "Redundance Switch“) automatisch lückenlos nachlesen. Auch die Auskopplung von Meldearchiven ist auf diesem Weg ausfallsicher möglich. Eine derartige Umsetzung wurde unter dem Namen „KSHistBuilder" als PIMS-unabhängige Lösung entwickelt und in zahlreichen industriellen Installationen erfolgreich eingesetzt (siehe Bild 5).

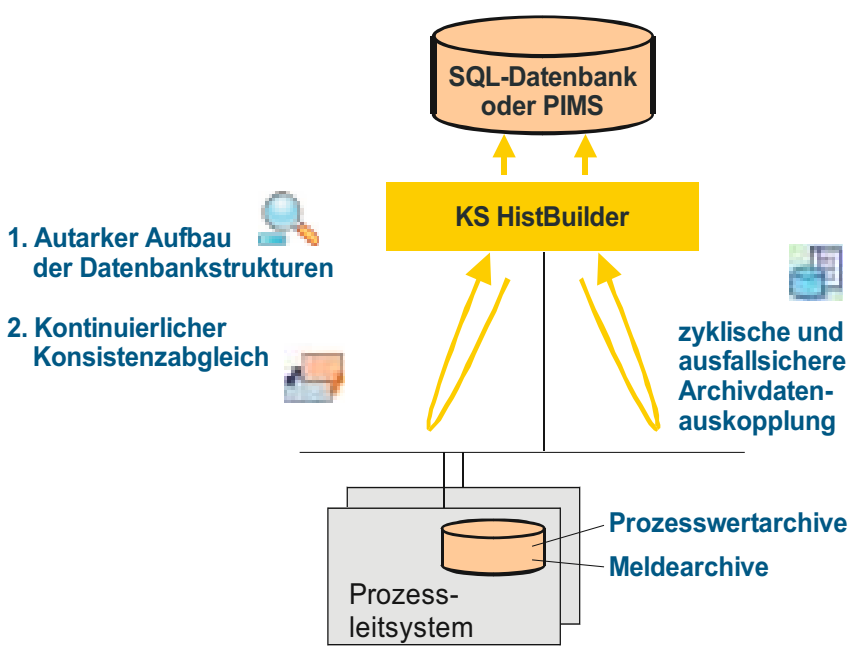

Bild 5: Selbstkonfiguration und automatisiertes Änderungsmanagement einer zentralisierten Betriebsdatenerfassung.

Mit dieser Strategie wird das Leitsystem zur "Single Source of Engineering". Eine zentralisierte Betriebsdatenerfassung kann im vollautomatischen Modus ohne jegliche datenpunktspezifische Konfigurationen auskommen. Dabei werden sämtliche im Leitsystem archivierten Zeitreihen komplett übernommen und damit auch deren äquidistanten oder nichtäquidistanten Abspeicherungsvorgaben.

Einwände gegen diese Strategie betreffen insbesondere den Speicherumfang (nicht alle im Leitsystem archivierten Messstellen werden im PIMS benötigt) und den Bedarf gesonderter Komprimierungsvorgaben. Diese Einwände stammen noch aus der Motivation, Speicherplatz aus Kostengründen einzusparen. Dieser Aspekt verliert jedoch an Bedeutung, da Aufwendungen eines doppelten aber vermeidbaren Engineerings höher anzusetzen sind als Hardwareinvestitionen in Datenspeicher. Praxiseinsätze haben gezeigt, dass mit dem beschriebenen Lösungsansatz eine vollautomatische Einrichtung und Konsistenzsicherung zentralisierter Betriebsdatenerfassungen möglich ist. Es sind bereits zahlreiche Referenzanwendungen in Unternehmen der Grundstoffindustrie, Lebensmittelindustrie und Chemie erfolgreich umgesetzt. Der Einsatz eines Engineering- und Nutzdatenkopplers, der nach dem beschriebenen Prinzip arbeitet, ist sowohl in Verbindung mit gängigen PIMS-Systemen als auch in Verbindung mit offenen Standard-SQLDatenbanken möglich.

\subsection{Asset- und Instandhaltungsmanagement}

Anlagennahes Asset Management basiert auf den vermehrt zur Verfügung stehenden Parametrierungs- und Diagnosedaten von Geräten und Systemkomponenten. Ein Anwendungsfall ist die Überwachung von Systemressourcen wie CPU-Auslastung oder Speicherkapazität (s. [17]). Für Anlagenbetreiber ist es von Bedeutung, Hinweise über drohende Systemüberlastungen zu bekommen, um Ausfälle wichtiger Systemkomponenten zu verhindern. Möchte man eine derartige Überwachung zentralisieren, um z.B. betriebs- oder standortweit sämtliche Systemkomponenten aller Leitsys- 


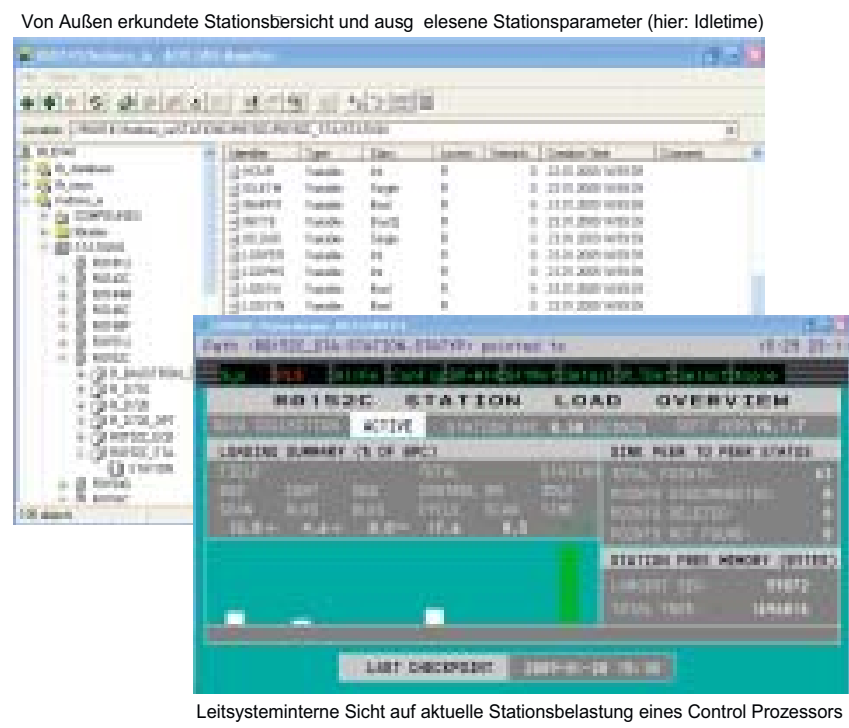

Bild 6: Offene Erkundung von Systemkomponenten als Basis zur konfigurationslosen Systemressourcenüberwachung.

Tabelle 1: Typische gleichartige Datenobjektstrukturen in Leitsystemen.

\begin{tabular}{|c|c|c|}
\hline $\begin{array}{l}\text { Applikations- } \\
\text { feld }\end{array}$ & $\begin{array}{l}\text { Basisdaten- } \\
\text { objekte }\end{array}$ & $\begin{array}{l}\text { Typische } \\
\text { Datenobjektstrukturen }\end{array}$ \\
\hline $\begin{array}{l}\text { Benutzerober- } \\
\text { flächen }\end{array}$ & Bilder & $\begin{array}{l}\text { enthalten Bildobjekte } \\
\text { Bildobjekte sind typisiert } \\
\text { Bildobjekte besitzen Parameter } \\
\text { Bildobjekte können Bildobjekte enthal- } \\
\text { ten } \\
\text { Bildobjekte können miteinander in Be- } \\
\text { ziehung stehen }\end{array}$ \\
\hline Schaltlogiken & CFC-Pläne & $\begin{array}{l}\text { enthalten Funktionsbausteine } \\
\text { Funktionsbausteine sind typisiert } \\
\text { Funktionsbausteine besitzen Parameter } \\
\text { Funktionsbausteine können Funktions- } \\
\text { bausteine enthalten } \\
\text { Funktionsbausteine können miteinander } \\
\text { in Beziehung stehen }\end{array}$ \\
\hline $\begin{array}{l}\text { Ablaufsteue- } \\
\text { rungen }\end{array}$ & SFC-Pläne & $\begin{array}{l}\text { enthalten Schritte und Transitionen } \\
\text { Schritte und Transitionen besitzen Para- } \\
\text { meter } \\
\text { Schritte können Schritte enthalten } \\
\text { Schritte und Transitionen können mitein- } \\
\text { ander in Beziehung stehen }\end{array}$ \\
\hline $\begin{array}{l}\text { Batch- } \\
\text { steuerungen }\end{array}$ & Rezepte & $\begin{array}{l}\text { enthalten Rezeptelemente } \\
\text { Rezeptelemente sind typisiert } \\
\text { Rezeptelemente besitzen Parameter } \\
\text { Rezeptelemente können Rezeptelemen- } \\
\text { te enthalten } \\
\text { Rezeptelemente können miteinander in } \\
\text { Beziehung stehen }\end{array}$ \\
\hline
\end{tabular}

teme zu beobachten, bieten sich selbstkonfigurierende Monitoringlösungen an, die zunächst die Systemtopologie verteilter Systeme erkunden können, um dann pro gefundener Systemkomponente die Ressourcendaten auszulesen. Eine Topologieerkundung von Feldgeräten kann heute bereits konfigurationslos über Feldbus-Informationsserver, wie der Asset Management Box [5], realisiert werden. Die Erkundung von Leitsystemkomponenten, wie Applikationsserver oder Controller, kann über offene Schnittstellen, wie z.B. den ACPLT/KS Schnittstellen, oder OPC UA erfolgen. In
Bild 6 ist unten ein Screenshot eines Leitsystem Faceplates und oben ein zugehöriger Screenshot eines leitsystemunabhängigen Systembrowsers dargestellt. Es demonstriert, wie aktuelle Systemressourceninformationen, die intern im Leitsystem angeboten werden, auch über offene Netzwerkanwendungen automatisch gefunden und ausgelesen werden können. Bei zyklischer Auslesung dieser Daten können selbstkonfigurierende Anwendungen zur statistischen Auswertung oder auch zeitnah arbeitende Applikationen, die Darstellungen oder Mitteilungen über herannahende Probleme für Betriebsbetreuer anbieten, eingesetzt werden.

Bild 6 zeigt konkret eine ohne Vorabwissen gescannte Topologiestruktur und auslesbare Ressourcenparameter mehrerer Foxboro I/A Series Leitsysteme an einem Standort. Mit der verwendeten offenen Schnittstelle ist es möglich, konfigurationsfreie Überwachungsanwendungen aufzusetzen, die in kurzen Zyklen (z. B. alle $10 \mathrm{~min}$ ) die Systemparameter einlesen und auswerten und in längeren Zyklen die von ihr zu überwachenden Systemkomponenten neu einscannen, so dass die reale Systemlandschaft immer automatisch in der Asset Management Anwendung nachgehalten wird. Die INEOS Köln GmbH nutzt beispielsweise eine derartige Anwendung zur Überwachung ihrer Leitsysteme am gesamten Standort.

\subsection{Validierung von Leitsystemapplikationen}

Eine retrospektive Validierung von Leitsystemen nutzt PLTspezifische Daten zum Nachweis der Übereinstimmung von Anforderungsspezifikationen wie Lasten- und Pflichtenheften, Projektierungsrichtlinien bis hin zu GMP-Anforderungen. Best Practice Empfehlungen dazu finden sich u.a. in [18]. Für die spezifischen Anforderungen in der Pharmaindustrie existiert der Good Automated Manufacturing Practice Supplier Guide zur Verifikation von Automatisierungssystemen [19]. Die Vorgaben von GAMP sind nicht nur für die Pharmaindustrie relevant. Viele Aspekte dieser Richtlinien sowie darüber hinausgehende Engineeringüberprüfungen leittechnischer Applikationssoftware sind auch für nicht regulierte Branchen sinnvoll.

Voraussetzung für eine automatisierte retrospektive Validierung leittechnischer Applikationssoftware sind ebenfalls nach außen erkennbare und damit über geeignete Schnittstellen erkundbare Softwarestrukturen mit ausgezeichneten Datenobjekttypen und Eigenschaften. Vorteil der Leitsysteme ist, dass viele herstellerübergreifende vereinheitlichte Grundstrukturen vorzufinden sind. Applikationssoftware wird in Leitsystemen überwiegend konfiguriert und nicht ausprogrammiert. Die Strukturen unterliegen in vielen Applikationsfeldern einem ähnlichen Datenmodell, basierend auf typisierten Datenobjekten und Datenobjektbeziehungen (siehe Tabelle 1).

Die letzte Spalte in Tabelle 1 zeigt anhand der gleichartigen Formulierungen, dass leittechnische Software unabhängig vom Applikationsfeld einem einheitlichen Grundmuster gehorcht. Selbstkonfigurierende Verfikationsanwendungen können diese gleichartigen Strukturen für vielfältige Anwendungen nutzen, die drei grundlegenden Gebieten zugeordnet werden können: 


\subsubsection{Kontinuierliche Änderungskontrollen („Management of Change")}

Die Vorgabe hierfür ist, dass einmal geprüfte Applikationsmodule unverändert bleiben oder Änderungen z.B. in Schichtbüchern bzw. in Melde- und Verriegelungslisten automatisch dokumentiert werden sollen. Hierfür ist eine laufende Kontrolle in dem Fall nötig, wenn sich ein expliziter Änderungsschutz im Prozessleitsystem nicht einstellen lässt oder Änderungen zwar möglich, aber Verantwortlichen angezeigt werden sollen. Beispiele für den zuletzt genannten Fall sind Deaktivierungen von Alarmierungen oder Regelungen, die von Anlagenfahrern auf Handbetrieb gestellt werden. Für kontinuierliche Änderungskontrollen können automatisch generierte Schichtbücher genutzt werden, die selbstkonfigurierend besonders wichtige Parameter, die man über spezifische Eigenschaften identifizieren kann, finden, um daraufhin deren Werte zyklisch auszulesen und erkannte Änderungen in einem Bericht oder in einer Visualisierungsoberfläche anzuzeigen.

\subsubsection{Parametrische Soll-Ist-Vergleiche}

In unternehmensinternen Anforderungskatalogen für Prozessleitsysteme wie Lasten- bzw. Pflichtenheften oder Projektierungsrichtlinien wird oftmals festgelegt, wie die Werte für bestimmte Controlparameter betriebsweit ausgelegt werden sollen. Relevant sind diese Festlegungen beispielsweise für Optionsparameter von Regler-, Motor- oder Ventilsteuerblöcken, die einheitlich festgelegt werden sollen, um betriebsoder unternehmensweit verlässliche Verhaltensweisen dieser Funktionsblöcke zu erhalten. Bei Abnahmetests oder bei kontinuierlichen (Routine-)Überprüfungen können selbstkonfigurierende Lösungen eingesetzt werden. Diese suchen vor jeder Untersuchungssequenz zunächst nach relevanten Bausteinen (z.B. Motorsteuerbausteine), um dann die aktuellen Werte der Optionseinstellungen auszulesen und gegen die pro Optionsparametertyp abgelegten Sollvorgaben zu prüfen. Die Sollvorgabenüberprüfung muss dazu oftmals betriebszustandsabhängig durchgeführt werden.

\subsubsection{Strukturelle Soll-Ist-Vergleiche}

Neben parametrischen Soll-Ist-Vergleichen kann eine Leitsystemverifikation auch durch strukturelle Soll-Ist-Vergleiche unterstützt werden. Hier werden Beziehungsstrukturen von Datenobjekten überprüft. Bei hierarchisch organisierten Leitsystemstrukturen können Vorgaben auf Begrenzung von Hierarchietiefen verifiziert, bei netzartig organisierten Datenobjektbeziehungen können ggf. nicht gewollte oder zwingend benötigte Rückschlüsse detektiert werden. Ein Beispiel ist die Organi- sation von Bedienbildern. Ergonomische Vorgaben können aussagen, dass eine maximale Bildhierarchietiefe (z. B.: Übersicht, Teilübersicht, Detail, Faceplate) vorzusehen ist. Jede Anwahl eines Bildes muss zudem die Möglichkeit bieten, wieder von diesem Bild weiter navigieren zu können, zumindest zurück zum Vorgängerbild. Diese Vorgaben bei Abnahmetests oder während des Lifecycles eines Anlagenbetriebs zu kontrollieren, ist sehr aufwändig.

Zur Verifikation und strukturellen Rückdokumentation von Operatorbildern kann eine selbstkonfigurierende Qualifizierungslösung eingesetzt werden. Bild 7 zeigt ein Beispiel eines automatisch generierten Erkundungsergebnisses in Form eines Graphen, der in diesem Fall die Verlinkung von Visualisierungsbildern eines PCS7-Systems zeigt. Die Applikation sucht eigenständig Bilder und in diesen nach Bildobjekten vom Typ „Button“. Ist ein derartiges Bildobjekt gefunden worden, werden über seine Clickeigenschaften Referenzierungen zu anderen Bildern herausgelesen. Die so gewonnenen Beziehungsstrukturen können dann in Form eines gerichteten Graphen visualisiert und mit Hilfe von Algorithmen auf Basis der Graphentheorie ausgewertet werden. Der Graph dient dazu, Bildabhängigkeiten gegen vorab formulierte strukturelle Vorgaben zu prüfen, unsinnige oder fehlende Verlinkungen zu detektieren oder auch ein ReEngineering von Bildern durch übersichtliche Darstellung der Zusammenhänge zu erleichtern.

Bild 7 zeigt einen Ausschnitt einer Graphenvisualisierung sämtlicher Leitsystembilder der Abflusssteuerzentrale der Stadtentwässerungsbetriebe der Stadt Köln. In der Abflusssteuerzentrale werden ca. 200 über das gesamte Stadtgebiet von Köln verteilte Bauwerke (Pumpstationen, Schieber, Regenüberlaufbecken, ...) überwacht. Dazu wurden von unterschiedlichen Systemintegratoren ca. 370 Bilder mit über 600 Bildreferenzierungen im Leitsystem konfiguriert. In Bild 7 oben ist ein typisches Bedienbild dargestellt. Wie aus
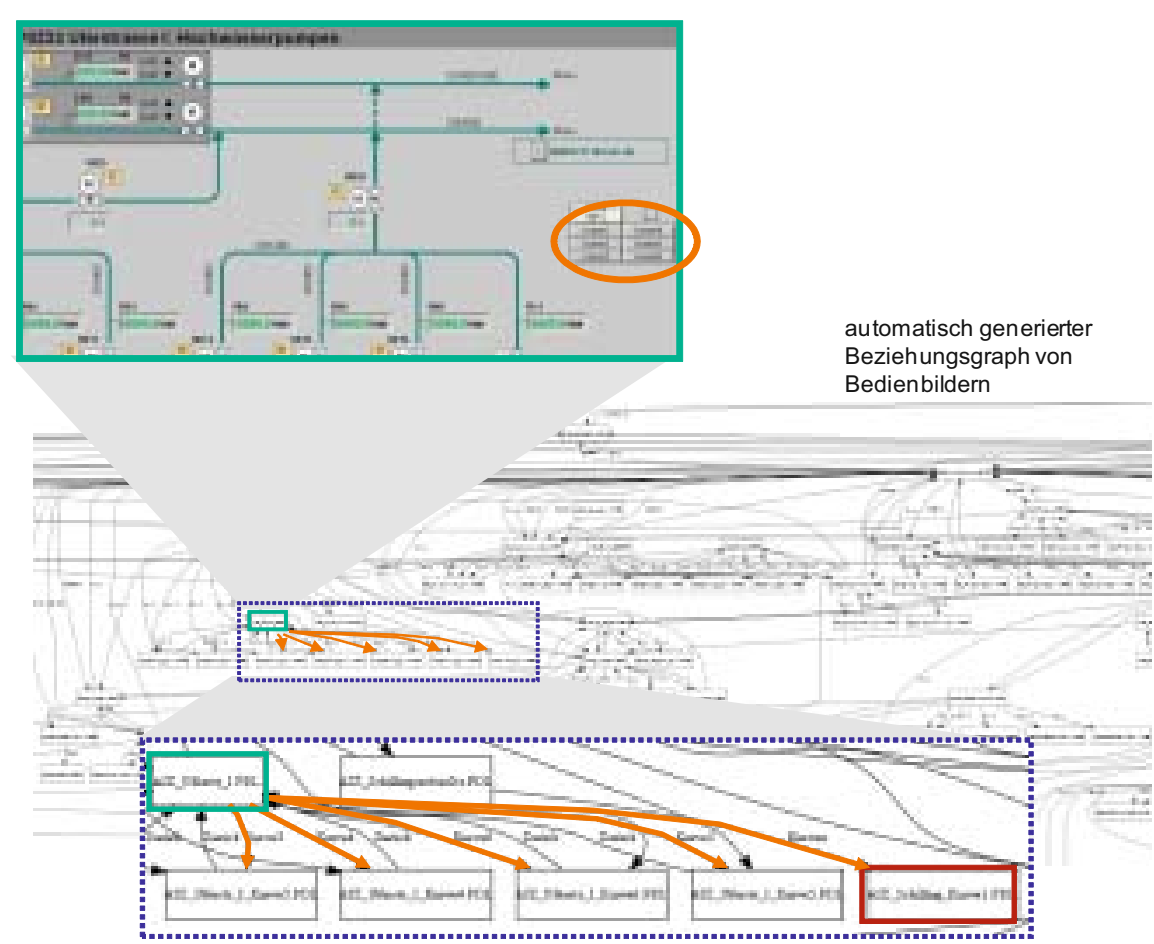

Bild 7: Automatisch generierter Graf aller Bildabhängigkeiten eines PCS7-Systems. 
dem automatisch generierten Beziehungsgraphen aller Bildabhängigkeiten ersichtlich ist, ist dieses Bild (im Graph als grün umrandeter Knoten herausgestellt) in ein Geflecht von Aufrufmöglichkeiten aus anderen Bildern eingebunden und stellt selber wieder den Ausgangspunkt zum Aufruf anderer Bilder dar. Die Schaltflächen „Kurve1" bis „Kurve6" (Steuerelementgruppe rechts im Bedienbild) werden im Graphen als Kanten zu anderen Bedienbildern repräsentiert. Mit Hilfe der automatischen Bilderkundung und graphenbasierten Aufbereitung konnten folgende Auswertungen zur Leitsystemverifikation durchgeführt werden:

- Automatisiertes Suchen von Bildern, zu denen hin referenziert wird, von denen aber bisher kein Bildsprung zu einem anderen Bild eingerichtet wurde (siehe rote Kennzeichnung in Bild 7). Graphentheoretisch wird nach Blättern gesucht, die im gerichteten Graf „Sackgassen“ bilden.

- Automatisierter Abgleich der im Graphen vorhandenen Knotennamen mit den im Leitsystem vorhandenen Bilddateien. So kann schnell herausgefunden werden, in welchen Bildern eine Navigationsmöglichkeit auf ein anderes Bild implementiert wurde, obwohl dieses Bild im Leitsystem noch gar nicht eingerichtet wurde.

- Visuelle Durchsicht der Graphendarstellung hinsichtlich einer syntaktischen Prüfung, ob sinnvolle Namen für die Bilder und deren Navigationsbuttons gewählt wurden.

Neben Qualifizierungsmaßnahmen können auch BulkEngineeringtätigkeiten durchgeführt werden. So können über Regeln die Default-Zeiträume von anzuzeigenden Trendkurven automatisch gesetzt werden (in der Abwassertechnologie typischerweise Tagesganglinien) oder auch Messbereichsvorgaben regelbasiert gesetzt werden. Voraussetzung dazu sind zum einen jedoch geeignet ausgelegte Anlagenkennzeichnungssysteme und zum anderen Schnittstellen mit Schreibfunktionalität für Parameterwerte (hier Zeit- und Messbereich von Trendkurvenobjekten).

Es sei angemerkt, dass für validierungspflichtige Anwendungen ein nachweislich fehlerfreies Vorwärtsengineering, das eine eindeutige Umsetzung von Planungsvorgaben (Bestandteil von CAE-Systemen) in Leitsystemstrukturen
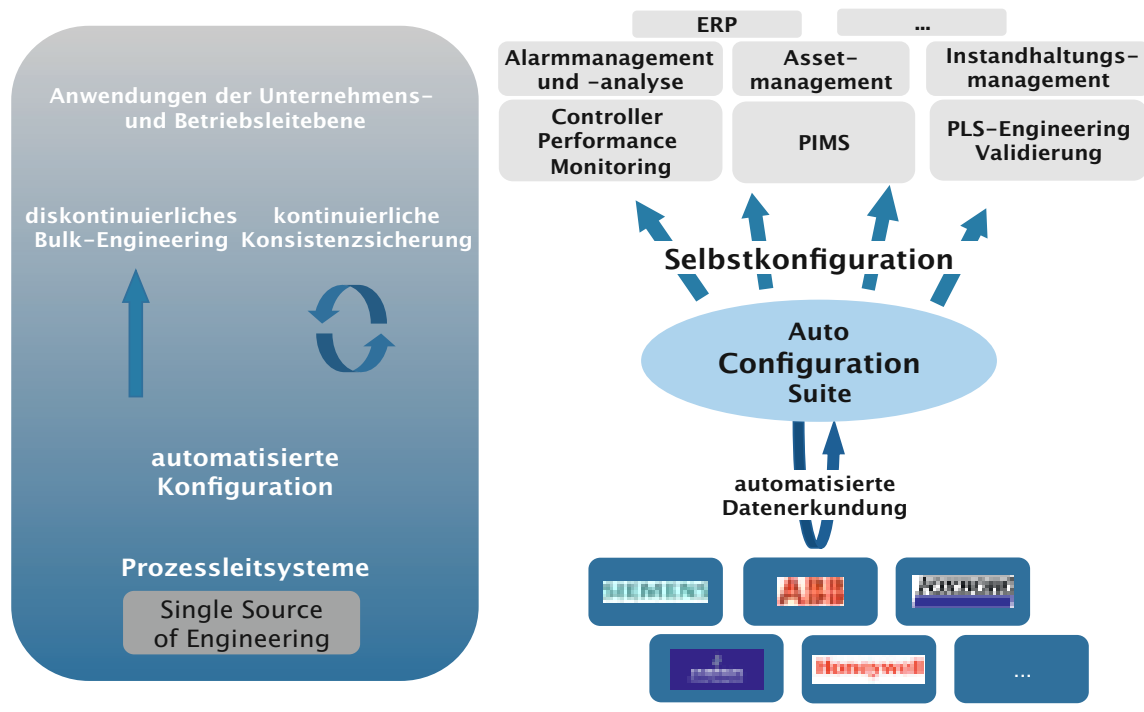

sicherstellt, immer der zu favorisierende Weg sein sollte (s. dazu [20]). Überall dort, wo aber dieser Weg nicht verfügbar ist, kann mit dem hier beschriebenen Ansatz der Aufwand für den dann nötigen retrospektiven Nachweis erheblich gesenkt werden.

\section{Generischer Lösungsansatz zur Selbstkonfiguration von MES-Lösungen}

Die oben dargestellten und in der Praxis eingesetzten Beispiele für selbstkonfigurierende Anwendungen zeigen, dass immer wieder gleichartige Mechanismen zur Umsetzung von Suchregeln genutzt werden. Daher ist es möglich, einen generischen Lösungsansatz zur Selbstkonfiguration von MES-Lösungen in Form einer "Auto Configuration Suite“ bereitzustellen.

Die in der Suite genutzten Mechanismen basieren dabei auf den folgenden gleichartigen Strukturen:

- Es werden Leitsystemschnittstellen genutzt, über die man die Datenhaushalte der Leitsysteme erkunden kann. Dabei können nicht nur hierarchisch strukturierte Datenobjektabhängigkeiten, sondern auch Querbeziehungen transparent abgebildet werden und somit von externen Konfigurationsklienten netzartige Suchpfade dynamisch durchlaufen werden.

- Es können pro Datenobjekt einheitlich festgelegte Metainformationen (u.a. „Typ eines Datenobjektes") gesucht und ausgelesen werden. Damit lassen sich systemunabhängige Regeln und Filterangaben für Suchanfragen formulieren.

- Die Übernahme der Engineeringdaten erfolgt je nach Systemanforderungen entweder einmalig als automatisch generiertes Bulk-Engineering oder im kontinuierlichen Betrieb, so dass eine laufende Konsistenzsicherung zwischen den Konfigurationsdaten des Leitsystems und der überlagerten Anwendung über den gesamten Lebenszyklus von Systemkopplungen gewährleistet wird.

- Die Ergebnisse der Engineeringdatensuche werden immer zunächst in Form einer vereinheitlichten XMLStruktur abgebildet. Für ein Bulk-Engineering können diese Daten mit Hilfe von XML-Stylesheets in unterschiedliche Syntaxformate (z.B. systemspezifische csv-Dateiformate) umgewandelt werden. Die Stylesheets können in Bibliotheken abgelegt werden, so dass für marktgängige MES-Lösungen diese Stylesheets ohne eigenen Programmieraufwand zur Verfügung stehen.

Die Auto Configuration Suite kann damit als generisches Framework für einmalige oder laufende Engineeringdatenauskopplungen genutzt werden. Alle oben beschriebenen Anwendungsfälle selbstkonfigurierender Systeme können damit abgedeckt wer-

Bild 8: Auto Configuration Suite. 
den. Die Suite kann als ergänzende Lösung zu bestehenden MES-Anwendungen sowohl von Systemanbietern, Systemintegratoren als auch von Anlagenbetreibern genutzt oder in vorhandene MES-Systeme integriert werden. Bei der BASF wurde mit Hilfe der Auto Configuration Suite eine erste Anwendung zur automatischen Erzeugung von Bulk Engineering Dateien für ein zentralisiertes Betriebsdatenerfassungssystem eingeführt.

\section{Literatur}

[1] Polke, M.: Prozessleittechnik. Oldenbourg Verlag München, 1994.

[2] IEC: IEC 62264 Enterprise Control System Integration, 2003 (als Deutsche Norm: DIN EN 62264 Integration von Unternehmensführungsund Leitsystemen, 2008).

[3] Enste, U., Müller J.: Datenkommunikation in der Prozessindustrie. Oldenbourg Industrieverlag, München, 2007.

[4] Kunze, C.: Ubiquitous Healthcare: Anwendung ubiquitärer Informationstechnologien im Telemonitoring. Dissertation Universität Fridericiana Karlsruhe, 2005.

[5] Müller, J.: Automatisierung des Anlagennahen Asset Managements in der Prozessindustrie. Dissertation Lehrstuhl für Prozessleittechnik, RWTH Aachen, 2007.

[6] Fedai, M., Drath R.: CAEX - ein neutrales Datenaustauschformat für Anlagendaten - Teil 1 und Teil 2. atp - Automatisierungstechnische Praxis (2004), Oldenbourg Inustrieverlag, München.

\section{Fazit}

Um weitere Wertschöpfungspotentiale von Produktionsprozessen und der Anlagenbewirtschaftung zu nutzen, bietet es sich an, ergänzende IT-Lösungen zur Produktivitätsanalyse und -optimierung bzw. der Weiterverarbeitung von Produktionsinformationen einzusetzen. Ein Hindernis ist aber momentan noch der hohe Aufwand für deren Inbetriebnahme und dauernden Konfigurationspflege. Die Automatisierung von Konfigurationsvorgängen verteilter Systeme stellt daher einen wichtigen Schritt dar, um den vermehrten Einsatz hilfreicher Zusatzanwendungen zu unterstützen.

In diesem Artikel wurden Randbedingungen, Umsetzungsvorschläge und Anwendungsbeispiele aus dem Bereich der Prozessleittechnik beschrieben. Dabei wurde der Fokus auf die Selbstkonfiguration von MES-Lösungen auf Basis erkundbarer Leitsystemengineeringdaten gelegt. Die beschriebene Herangehensweise gilt in gleicher Form aber auch zur automatisierten Ankopplung anderer Systeme, wie CAE oder ERP-Systeme. Auf Basis verallgemeinerter Strukturen wurde schließlich ein generisches Framework vorgestellt, das zur Umsetzung automatisierter Engineeringdatenauskopplungen genutzt werden kann.

Die beschriebenen grundlegenden Ideen der Nutzung vereinheitlichter Systemerkundungsmöglichkeiten können zukünftig in weiteren Anwendungsszenarien genutzt werden. Anwender und Systemhersteller sind daher eingeladen, ihre Interfacetechnologien in diese Richtung hin zu überprüfen und Selbstkonfigurationsmöglichkeiten auszuloten.
[7] T. Schmidberger, A. Fay, R. Drath, A. Horch: Von Anlagenstrukturinformationen automatisch zum Asset Management. atp - Automatisierungstechnische Praxis 06/2006, Oldenbourg Inustrieverlag.

[8] Ebel, M.: Automatisiertes Leitsystem-Engineering mittels CAEX, visIT, 2/2007, Fraunhofer IITB, 2007.

[9] Brecher, C. et. al.., Plug\&Play“ - eine Vision rückt näher. A\&D-Kompendium 2008/2009, publish-industry Verlag, München, 2008.

[10] Schmitz S., Epple, U.: Automatisierte Projektierung von HMI-Oberflächen, in VDI-Berichte 1980, GMA Kongress 2007.

[11] Epple, U.: Austausch von Anlagenplanungsdaten auf der Grundlage von Metamodellen. atp - Automatisierungstechnische Praxis, 45 (2003) 7, Oldenbourg Inustrieverlag, München, S. 61-70.

[12] Albrecht, $H$.: On Meta-Modeling for Communication in Operational Process Control Engineering. Dissertation. VDI Fortschritt-Bericht, Series 8, No. 975, VDI-Verlag, Düsseldorf, Germany.

[13] Lehrstuhl für Prozessleittechnik, RWTH Aachen: www.acplt.org.

[14] OPC Foundation: www.opcfoundation.org.

[15] NAMUR: Alarmmanagement. Namur Arbeitsblatt 102, 2008.

[16] EEMUA: Alarm Systems: A Guide to Design, Management and Procurement. EEMUA Publication No. 191, 1999.

[17] Lotz, P.: Zustandsüberwachung und Diagnose von Prozessleitsystemen. atp - Automatisierungstechnische Praxis 04/2009, Oldenbourg Inustrieverlag, München.

[18] VDI VDE Richtlinie: Validierung in der Prozessleittechnik. Beuth Verlag, Berlin, 2003.

[19] Good Automated Manufacturing Practice Forum: GAMP 5, 2008.

[20] Tauchnitz, T.: CIPE oder: Die Zeit ist reif für eine Integration des Prozessentwurfs-, Engineering- und Betreuungs- Prozesses - Teil 1 und Teil 2. atp - Automatisierungstechnische Praxis 10 und 11/2005, Oldenbourg Inustrieverlag, München.

Manuskripteingang: 11.2.2009

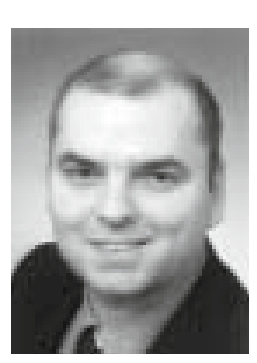

Dipl.-Ing. Senad Bukva (30) ist seit 2008 Mitarbeiter der LeiKon $\mathrm{GmbH}$, Herzogenrath. Er ist als Projektleiter verantwortlich für Systementwicklungen und Inbetriebnahmen von Lösungen des betrieblichen Informationsmanagements.

Adresse: LeiKon GmbH, Kaiserstr. 100, D-52134 Herzogenrath, E-Mail: senad.bukva@leikon.de

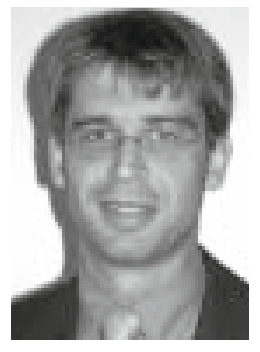

Dr.-Ing. Udo Enste (40) ist seit 2000 Geschäftsführer der LeiKon $\mathrm{GmbH}$, Herzogenrath. Seine Hauptarbeitsgebiete liegen in den Bereichen Planung und Konzeption von Leit- und MES-Systemen, industrielle Datenkommunikation, betriebliches Informationsmanagement und Effizienzanalysen und -optimierungen. Er ist Mitglied im DIN DKE-Komitee 931 'Systemaspekte in der Leittechnik' sowie im VD GMA-Fachausschuß, Industrielle Anwendung komplexer und adaptiver Regelungen' und Dozent am Haus der Technik, Essen.

Adresse: LeiKon GmbH, Kaiserstr. 100, D-52134 Herzogenrath, E-Mail: udo.enste@leikon.de

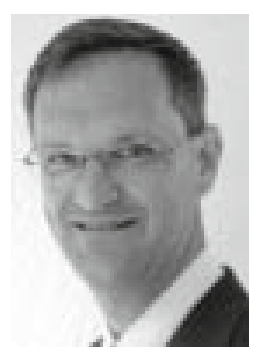

Dr.-Ing. Felix Uecker (39) ist seit 2000 Geschäftsführer der LeiKon $\mathrm{GmbH}$, Herzogenrath. Seine Hauptarbeitsgebiete liegen in den Bereichen Planung und Engineering von Leit- und Betriebsdatenerfassungssystemen, betriebliches Informationsmanagement und Asset Management. Er ist Mitglied im VDI/VDE und in der DWA.

Adresse: LeiKon GmbH, Kaiserstr. 100, D-52134 Herzogenrath, E-Mail: felix.uecker@leikon.de 